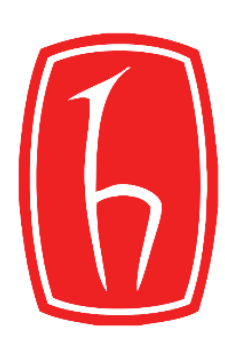

Hacettepe Üniversitesi Edebiyat Fakültesi Dergisi

Hacettepe University Journal of Faculty of Letters

Cilt/Volume: 35 Sayl/Number:2

Aralı/December 2018

doi: 10.32600/huefd.432238

\title{
Fehleranalyse bei der Übersetzung von idiomatischen Wendungen in dem Werk "72. Zelle” von Orhan Kemal*
}

\author{
An Analysis of the Translation Errors of Idioms and Proverbs in the work "Ward 72" by \\ Orhan Kemal
}

Yüksel BAŞARMIŞ ${ }^{* *}$

\begin{abstract}
Öz
Kalıplaşmış dil birimlerinin hedef dile aktarılması, edebi eserlerin başka bir dile çevirisi sürecinde çevirmenin karşılaşı başlıca sorunlardan biridir. Kaynak dil ile hedef dil arasındaki dilsel ve kültürel farklılıklar arttıkça, esas görevi kaynak dildeki eserin okuyucu üzerinde bıraktığı etkiye, hedef dilde de ulaşması beklenen çevirmenin işinin de zorlaştığı bilinmektedir. Atasözü ve deyimlerin çevirisinde eşdeğerliliğin ne kadar sağlanabildiği, çevirmenin bu zorlukları aşmak için hangi yöntemleri tercih ettiği ve ne tür hataların yapıldığı, hatalara neden olan faktörlerin neler olduğu, bu çalışmada incelenecek konulardır. Kalıplaşmış dil birimlerinin özellikleri ve hedef dilde bire bir karşılıklarının bulunmadığı durumlarda izlenecek yöntemler irdelenecek, çevirmenin aktarımlarında izlediği hatalı tutumlar ve hataların önlenebilirliği üzerinde durulacaktır. Konu ile ilişkili olarak bir çevirmenin yeterliliklerinin neler olması gerektiği ve kendisinden nelerin beklendiği araştırılarak çevirmen profilinin incelenmesine de yer verilecektir. "72. Koğuş" adlı eserin Almancaya çevirisini yapan Achim Martin Wensien' in ("Die 72. Zelle") mesleki kariyeri üzerinde durulması da gerekli görülmüştür. Türk Edebiyatının usta kalemlerinden olan Orhan Kemal'in "72. Koğuş" adlı eserinde geçen atasözü ve deyimlerin oldukça yüksek bir frekansa sahip olması araştırma için önemli bir kaynak sağlamaktadır.
\end{abstract}

Anahtar sözcükler: Orhan Kemal, deyimler, atasözleri, çevrilebilirlik sorunu, çeviri hataları, çevirmen profili

\begin{abstract}
Translation of the idioms into the target language is one of the fundamental problems faced by translators in the process of translation of literary works into another language. It is known that as the linguistic and cultural differences between the source and target languages increase, the translator, whose main task is to reflect the effect of the work in its original language to the target language, faces more challenges. This study will analyze the equivalence of the translation of proverbs and idioms; techniques preferred by the translators to overcome the challenges and the types of errors made and the factors leading to the mistakes. The method to be followed in the case of language clichés and when no equivalents are found in the target language, the faulty strategies of the translator and preventing these mistakes, will be emphasized. In this context, the study will also cover the competence a translator should demonstrate and what is expected of him/her, along with the analysis of a translator's profile. The professional career of Achim Martin Wensien, the translator of "Ward 72/ "Die 72. Zelle" by Orhan Kemal will also be studied. The fact that "Ward 72 " by the renowned Turkish writer Orhan Kemal has a high frequency of proverbs and idioms provides a valuable source for this research.
\end{abstract}

Keywords: Orhan Kemal, Idioms, Proverbs, Translationability of phrases, Translation errors, Translator Profile

\footnotetext{
* Dieser Artikel ist aus der Masterarbeit „Das Problem der Übersetzbarkeit der Idiomatischen Ausdrücke in den Werken "72. Zelle" und "Murtaza" von Orhan Kemal." Von Yüksel Başarmış entstanden.

*** Yüksel Başarmış, Okutman, TOBB Universität, Fremdsprachenabteilung, ybasarmis@etu.edu.tr
} 


\section{Einleitung}

Für die vorliegende Arbeit wurde das Werk "72. Koğuş" von Orhan Kemal und die deutsche Übersetzung von Achim M. Wensien "Die 72. Zelle” gewählt. In Anlehnung an die Übersetzungstheorie von Werner Koller wird analysiert, welche Vorgehensweisen der Übersetzer des Werks "Die 72. Zelle“ bevorzugt hat und welche Beweggründe ihn zu seiner Entscheidung getrieben haben.

Die Grundlage zu der vorliegenden Analyse stellt die hohe Frequenz der Redewendungen und sprichwörtlichen Redensarten dar. Zu dem Sprachstil des Schriftstellers müssen an dieser Stelle einige hinweisende Vorbemerkungen gemacht werden. Das Gefängnis bzw. die Zelle, stellt den Handlungsrahmen des Werks dar und dokumentiert die soziale Schicht und das Bildungsniveau der Gestalten. Dies wird durch den Sprachgebrauch und durch die häufige Anwendung der kulturellen Elemente übermittelt. Musa Yaşar Sağlam (2014, s. 94) bemerkt hierzu, dass die Verwendung der Sprichwörter und Redewendungen in den Werken, in denen die Helden ein niedriges Bildungsniveau haben und die aus unteren sozialen Schichten stammen, relativ häufig vorkommen. Es lässt sich laut Sağlam (ebd. 2014) feststellen, dass die Menschen aus unteren, sozial schwachen und bildungsfernen Schichten stammen, einen begrenzten Sprachgebrauch haben und danach tendieren, ihre Gedanken mit Sprichwörtern und Redewendungen zum Ausdruck zu bringen. Der Grund zu dieser Tendenz ist ihre begrenzte sprachliche Kompetenz. Auf der anderen Seite signalisiert das häufige Verwenden dieser Kulturelemente die Ersparung eigener Denktätigkeit dieser Gestalten, denn sie selbst sind nicht in der Lage, eine eigene angemessene sprachliche Form zu entwickeln. Aus dem beschriebenen Umfeld stammen die Gestalten dieses Werks. Das Originalwerk 72. Koğuş besteht aus 96 Seiten, die Übersetzung aus 113 und das Originalwerk beinhaltet fast 326 Redewendungen und Sprichwörter. Die Frequenz der idiomatischen Wendungen in dem Werk ist $1.01 \%$ [Frequenz= Zahl der Seiten/Zahl der Idiome]. Der Übersetzer dieses Werks sendet folglich außerordentlich aufschlussreiche, lehrreiche Verhaltensbeispiele zum Übersetzungsverfahren.

Anhand dieses Werks und dessen Übersetzung wird versucht festzustellen, welche Verfahren der Übersetzer bevorzugt hat, und ob und wie weit es dem Übersetzer gelungen ist, die Sprichwörter und die Redewendungen, die von dem realistischen Schriftsteller Orhan Kemal reichlich verwendet worden sind, dem Zielsprachenleser zu vermitteln. Der Schwerpunkt wird jedoch auf der Analyse der Übersetzerfehler liegen.

\section{Das Selbstbild des Übersetzers}

Einen professionellen und erfahrenen Übersetzer zu uterscheiden von denen, die oftmals übersetzt haben, ohne in dieser Branche ausgebildet zu sein, von den Unerfahrenen; mit/ohne einer Ausbildung, von Neustartern, denjenigen, die diese Tätigkeit zum Geld verdienen, neben ihrem eigentlichen Beruf zusätzlich ausüben, von denen, die aus Leidenschaft übersetzen, oder von denen, die dazu gezwungen, beauftragt oder darum gebeten werden, weil sie einfach die Ausgangssprache beherrschen, von freien, angestellten Übersetzern oder Beamten, setzt eine Definition voraus, was eigentlich eine translatorische Kompetenz ist, welche Eigenschaften von einem Übersetzer zu erwarten oder zu verlangen sind.

“Translatorische Kompetenz ist jene Fähigkeit, die den Übersetzungsvorgang (translatorisches Handeln) ermöglicht und über das allgemeine Kommunikationsvermögen des Individuums einerseits sowie über bloße Fremdsprachenkenntnisse andererseits hinausgeht. Translatorisches Handeln bedeutet die Produktion eines Textes als Informationsangebot in der Zielsprache über einen Text, der als Informationsangebot in der Ausgangssprache vorliegt, unter Berücksichtigung des intendierten Rezipienten” (Sandrini, 1993, s.54).

Das Vorhandensein des „Wissens“ eines Übersetzers wird von Wolfram Wills (2004) als die bedeutendste Leistungskraft hervorgehoben. 
„Alles, was mit übersetzerischen Leistungen $\mathrm{zu}$ tun hat, gleichgültig, ob es sich um Problemlösungsstrategien oder um routunisierte Übersetzungstechniken handelt, ist Anwendung von erlerntem Wissen, das durch fremd- oder Selbstinstruktion erworben worden sein kann. Durch sein Wissen gewinnt der Übersetzer Einsichten in die verschiedensten Zusammenhänge, kann er Schlussforderungen ziehen, seine Strategien, Methoden und Techniken ökonomisch organisieren“ (s. 219).

Wills deutet auf die drei Grundlagen des Wissens hin, und zwar auf den Erwerb, auf die Strukturierung und auf den Abruf des Wissens. Der Übersetzer muss sein Wissen erweitern, sie organisieren und in angemessener Zeit danach greifen können. So kann er konstruktiv handeln. (ebd. 2004, s.219). Relevant ist auch das Kulturwissen des Übersetzers. „Das Wesen des Übersetzungsprozesses kann man nur verstehen, wenn man ihn in einen soziokulturellen Rahmen einbettet“ (ebd. 2004, s. 225).

Jirí Levý (2012) stellt zusammengefasst drei Forderungen an den Übersetzer und weist dem Erfassen der Vorlage den ersten Platz zu (vgl. s. 24). Levý setzt voraus, dass ein guter Übersetzer vor allem ein guter Leser ist, "denn der Weg zum Verständnis des künstlerischen Ganzen führt über das Erfassen der stilistischen und inhaltlichen Werte der einzelnen Sprachmittel und Teilmotive hinaus” (vgl. ebd. 2012, s. 28).

„Textverstehen kann damit verstanden werden als die Dekodierung von Kultur. Ob die Botschaft eines Textes in ihrer intendierten Form beim Empfänger ankommt, ist einerseits von ihrer Enkodierung durch den Produzenten und andererseits von der Dekodierung durch den Rezipienten abhängig. Dafür ist der Rückgriff auf kulturspezifisches außersprachliches (also Weltwissen) und sprachliches Wissen erforderlich“ (Hennecke, 2009).

In seiner Eröffnungsrede zum Fachsymposium vergleicht Heinrich Pfeiffer (1993) den Übersetzer mit einem Fährmann, der sein Boot bzw. die Personen von einem Ufer zum anderen eines Flusses übersetzt. Die Tätigkeit des Übersetzers sieht er ähnlich. Pfeiffer meint, dieser verbindet zwei Sprachkulturen und betont, dass der Übersetzer dafür „eine enorme geistige Kraft und Kenntnis der der fremden Kultur“ braucht (vgl. s. 5).

„Im Übersetzen werden Barrieren der Zeit und des Raumes überwunden. Dazu benötigt der Übersetzer Kenntnisse der Geschichte, der Sprache, der Riten, der Gefühle, der Institutionen etc. Nicht nur diese Kenntnis der Fakten und der Geschehnisse, die man von draußen erkennen kann, sondern auch die Kraft der Kultur und Sprache von innen, zu verstehen, was ein Autor gedacht, gesehen, gefühlt und erahnt hat, gehört dazu“ (ebd s. 5).

Nach Pfeiffer habe Herder dies „Einfühlung“ genannt. Er stellt aber schließlich fest, dass „diese Meßmethoden für den Übersetzer von keiner oder nur von begrenzter Bedeutung sein können“. Er kommt zu der Schlussfolgerung, dass der gute Übersetzer es wei $\beta$, den Gedanken und die Person des Autors uns vor Augen zu führen (vgl. ebd s. 6). Das Vertrauen an die eigene Intuition findet nach Jan Engberg bei Radegundis Stolze ihre Stimme.

„In der Theorie von Stolze spielen die Begriffe "Intuition” und "Stimmigkeit” eine große Rolle. Intuition wird als wesentlicher Teil des menschlichen Verstehens und somit als notwendiger Teil des Über-setzungsprozesses gesehen. Anhand des intuitiven Verständnisses der Textvorlage, das auf sein sprachliches und sonstiges Vorwissen baut, fertigt der Übersetzer einen Übersetzungsvorschlag an, der dann durch rationale Überlegungen nachgeprüft und gegebenenfalls geändert wird [...]. Intuition und damit verbunden auch Kreativität gehören also zu den bei jeder Übersetzung einzusetzenden Denkmethoden“ (Engberg, 1992, s. 110). 
Es ist fraglich, ob Übersetzer sich mit den Erwartungen, Anforderungen oder Theorien der Wissenschaftler beschäftigen oder sogar darüber informiert sind, was von ihnen gefordert wird, aber die Wissenschaft beschäftigt sich mit ihrem Tun und Handeln. Fest steht, dass man im Nachhinein nicht wissen kann, nach welchen Kriterien und aus welchen Gründen der Übersetzer eine Entscheidung getroffen hat, weil es sehr viele Einzelfälle zu treffen sind. Es lassen sich also oft nur Anmutungen erfassen.

\section{Achim Martin Wensien, der Übersetzer des Werks „72. Zelle“}

Wensien wurde 1951 in Anatolien, in der Türkei geboren und verbrachte Kindheit und Schulzeit in der Türkei. Seit 1970 lebt er in Deutschland, studierte in Deutschland Diplom-Soziologe und ist ausgebildeter Heimleiter in Altenhilfe. Wensien ist Autor und Werbetexter für Deutsch und Türkisch und beeidigter Dolmetscher und Übersetzer Deutsch -Türkisch.

Neben der Buchübersetzung von Orhan Kemal „Die 72. Zelle“ hat Wensien ein Buch namens „Der Mann mit dem Filzhut und Kaschmirmantel, Eine Saison mit Orhan Kemal“ verfasst.

Uli Rothfuss (2005) äußert eine Kritik über das Buch "Der Mann mit Filzhut und Kaschmirmantel" von Wensien und informiert kurz über den Inhalt des Buchs: Saffet Düzgün, der Held der Erzählung ist tief beindruckt von den Romanen Orhan Kemals und merkt, dass es in den Schilderungen der Romane und auch in der Biographie des bekannten Autors Ähnlichkeiten mit seinem eigenen Leben gibt.

Die Zeichen seines Aufenthaltes in der Türkei sind auch in seinem Buch „Die Eroberung des Maulbeerbaumes. Glückliche Kindheit in Anatolien“ zu sehen.

Ein Kontaktversuch mit Wensien blieb ohne Erfolg, zu den begrenzten Informationen konnte durch die Internetrecherche gelangt werden.

\section{Zur Übersetzung der idiomatischen Wendungen}

Auf eine Kategorisierung der Phraseologismen nach ihrem u.a. sprachlich/strukturellen, themenbezogenen Stellenwert oder auch nach ihren verschiedenen Merkmalen kann hier nicht eingegangen werden. Allgemein werden sie folgend definiert:

„Phraseologismen sind Wortverbindungen, die aus zwei oder mehr Wörtern bestehen. Die Wörter bilden eine durch die syntaktischen und semantischen Regularitäten der Verknüpfung nicht voll erklärbare Einheit. Sie sind idiomatisch, was bedeutet, dass die Bedeutung des Phraseologismus nicht als Summe der Bedeutungen der Einzelelemente beschreibbar ist“(Wordpress, Phraseologismen). „In lexikalischer Hinsicht sind Phraseologismen dadurch charakterisiert, da $\beta$ sie einerseits aus mehreren selbständigen Wörterbucheinheiten bestehen und andererseits selbst wieder eine lexematische Einheit bilden“ (Burger 1982, s. 2). Recep Özbay (2010) veranschaulicht den Unterschied zwischen den einfachen Wörtern und den Phraseologismen in seinem Beitrag „Äquivalenz bei türkischen und deutschen Somatismen mit „Hand“ und „Auge“ folgend: „Phraseologismen unterscheiden sich von einfachen Wörtern dadurch, dass sie strukturell aus mehreren Komponenten bestehen, aber wie bei einfachen Wörtern eine Gesamtbedeutung aufweisen, [...]“ (s.104). Er akzentuiert, dass sich die Gesamtbedeutung der idiomatischen Wendungen aus den einzelnen Wörtern nicht zu erschließen sind (ebd. s. 104). Die Wendungen „einen Kater haben“ und „Schwein haben“ liefern informative Beispiele in diesem Sinn. „einen Kater haben“ kann wörtlich bedeuten, dass man eine männliche Katze hat, diese Wendung ist in diesem Gebrauch keine feste Verbindung. Das Wort „Kater“ kann z.B. durch „einen Hund“ ausgetauscht werden. Aber wenn man die mataphorische Bedeutung „betrunken sein“ meint, kommt ein Austausch der lexikalischen Elemente nicht in Frage. In diesem Beispiel hat die Wendung durch Idiomatizität gekennzeichnete Gesamtbedeutung wie im Beispiel 
„Schwein haben“, was „Glück haben“ bedeutet. Diese Komponente lassen sich weder austauschen, noch durch andere Wörter ersetzen. Burger (1982) meint:

„Für die meisten Phraseologismen gilt aber folgendes: Ihre Gesamtbedeutung, die sie als lexikalisierte Einheit haben, entspricht nicht der Summe der Bedeutungen der einzelnen Wörter, aus denen sie bestehen. Die phraseologische Bedeutung ist verglichen mit der wörtlichen Bedeutung der Wortverbindung ein entweder spezielles oder anderes und neues Ganzes“ (s. 3).

„Wenn man berücksichtigt, dass die Wirkung der kulturellen Elemente auf die Leser der Originalsprache, die eine gemeinsame Sprache und die kulturellen Werte teilen differiert, dann kann man einsehen, dass der Literaturübersetzer öfters einer schwierigen Aufgabe sowie problematischen Äquivalenzanforderungen ausgesetzt ist“" (Başarmış, 2016, s. vii). Das Problem der Übersetzbarkeit der kulturellen oder idiomatischen Spracheinheiten tritt öfters dann auf, wenn sich in der Ausgangs- und der Zielsprache strukturell und kulturell weniger Ähnlichkeiten aufweisen oder sogar stark voneinander abweichen. Daher zählt die Erschaffung von konnotativer Äquvalenz „[...] zu den meist nur annäherungsweise lösbaren Problemen des Übersetzens; umso wichtiger sind korpusorientierte, sprachtextbezogene Analysen einzelner konnotativ "geladener" lexikalischer und syntagmatischer Bereiche“ (Koller, 2004, s. 241).

„Da man beim Übersetzen von Texten mit kontextualisierten Idiomen zu tun hat, ist zu erwarten, dass Kontextsensitivität und semantische Variabilität von Idiomen beim Übersetzen in besonderer Weise relevant werden und dass Übersetzungen selbst Hinweise auf eine semantische Variabilität eines ausgangssprachlichen Idioms liefern können“ (Sabban, 2010, s. 194).

Sabban fügt in ihrer Studie „Zur Übersetzung von Idiomen im Wörterbuch und im Text: die Rolle von Kontextsensitivität und semantischer Variabilität“ hinzu, dass Wörterbuchäquivalente nicht immer eine Lösung zur Übersetzung bieten, zumal das Finden dieser Elemente schon an sich für den Übersetzer ein Problem darstellen. Die Darbietung der Phraseologismen in Wörterbüchern bringt kein Ergebnis, weil Phraseologismen zum Teil aus Zusammensetzungen bestehen und oft mehrere Komponenten an ihrer Zusammensetzung vorweisen. Hier kommt man auf den Punkt: Welchen Mitteln bedient sich der Übersetzer?

„Die Recherchierkompetenz ist für jeden Übersetzer wichtig und will gelernt sein. Hier reicht es bei weitem nicht aus, nur Wörterbücher zu konsultieren, im Gegenteil, es sind andere Nachschlagwerkeheutzutage auch Abfragen im Internet, die dem Übersetzer das entsprechende fachliche Umfeld, u.a. in Form von Paralleltexten liefern“ (Simmonas, 2006, s. 66). Auf die Wichtigkeit der Recherchiertechnik als Hilfsmittel für den Übersetzer geht auch Christiane Nord ein und weist darauf hin, dass der Übersetzer diese beherrschen muss. Beim Prozess der Übersetzung sind vier Fertigkeiten gefragt. Diese sind: Auftragsanalyse, Ausgangstextanalyse, Strategieentwurf und Zieltextproduktion, wobei folgende Begriffe auf die Tätigkeiten beim Übersetzen die Zwischenphasen darstellen: Vergleich, Entscheidung, Umsetzung und Qualitätsicherung. Sie betont, dass der Übersetzer diese Fertigkeiten beherrschen muss:

,[...] die Fähigkeiten sich zu entscheiden, die Fähigkeit, die Ergebnisse von Übersetzungsauftragsanalyse und Ausgangstextanalyse so weit zu abstrahieren, dass man sie vergleichen kann, die Fähigkeit, für richtig befundene Strategieentscheidungen kreativ in sprachliches Handeln umzusetzen und schließlich die Fähigkeit, das eigene Produkt, den Zieltext, gegen den Übersetzungsauftrag zu halten und seine Qualität zu beurteilen“ (Nord, 2002, s. 79). 
Welche Verfahrensmöglichkeiten bleiben dann in diesem Fall dem Übersetzer noch vorhanden? Können Wissenschaftler oder Theoretiker den Übersetzern als Wegweiser behilflich sein? Lassen sich überhaupt Lösungsmethoden für Übersetzungsprobleme angeben?

Annette Kopetzki beschäftigt sich mit dieser Frage und liefert hierzu einen wichtigen Beitrag. Sie bemerkt, dass literarische Texte nicht nach Rezept „,decodiert“ werden und dass es keine allgemeinen Regel gibt, um sie ein für alle Mal richtig zu verstehen oder gar richtig zu übersetzen (vgl. Kopetzki, 2015, s. 77).

$\mathrm{Zu}$ betonen ist an dieser Stelle auch die Haltung der Übersetzer zu der Wisseschaft. „Von den allgemeinen Aussagen der Theorie wollen Übersetzer wenig wissen, vor allem, wenn die Wissenschaft ihnen Vorschriften macht. “ (ebd. 2015, s. 77). Kopetzki (2004) resümiert, dass Übersetzungsprobleme der Idiome meistens Einzelfälle sind und das Problem meistens im Verstehen der Idiome liegt. Nach Werner Koller (2011) ist es nicht das Anliegen der Übersetzungstheorie, anzuordnen, wie die Übersetzer zu übersetzen haben und nicht ,theoretische Konzeption als Richtschnur für ihre praktische Arbeit vorzugeben.“ (s. 13). Er äußert sich dazu folgend;

“ Vielmehr überliefern die Übersetzer mit ihren Übersetzungen, aber auch mit ihren Kommentaren zu ihrer Übersetzungsarbeit das empirische Material, das die Wissenschaftler analysieren, beschreiben und vielleicht sogar zu erklären versuchen. Zu hoffen ist, dass der Übersetzer wenigstens in einem Teil der Probleme, mit denen sich die Wissenschaft beschäftigt, seine eigenen erkennt, mit denen er es in seiner täglichen Praxis zu tun hat [...]” (ebd. s. 13).

Interessant ist die Äußerung von Lew Zybatow (2006), der die Aufmerksamkeit auf eine andere Perspektive lenkt. Er behauptet, dass nicht die Wissenschaft dem Übersetzer Methoden oder Theorien vorschlägt, sondern die Übersetzer mit ihren Verfahren der Wissenschaft Hinweise liefern.

„Es scheint in unserer jungen Disziplin (gemeint ist die Translationswissenschaft) noch keine hinreichende Klarheit über das Verhältnis zwischen Grundlagenforschung und angewandter Forschung bzw. zwischen Theorie und Praxis zu bestehen [...], weshalb es im Interesse einer erfolgreichen Entwicklung der Disziplin liegt zu begreifen, dass eine Wissenschaft in erster Linie für die Wissenschaft da ist“ (ebd. s. 156).

Betont wird, dass die Wissenschaft mehr von der Übersetzungspraxis des Übersetzers profitiert, als dass der Übersetzer einen Gewinn von der Wissenschaft zieht.

„Denn wenn die Translationswissenschaft sich selbst lediglich dadurch legitimieren will, den Praktikern oder Lehrenden gute Hinweise zu geben, wie sie zu übersetzen oder zu dolmetschen haben, dann beraubt sich die Translationswissenschaft selbst der Möglichkeit, ihren Gegenstand - die Translationwissenschaftlich zu klären, bevor sie darauf aufbauend praktische Methoden und Hinweise formulieren kann“ (ebd. 2006, s.156).

\section{Die Analyse der Übersetzungen der idiomatischen Wendungen in dem Werk „72. Zelle“}

Für Übersetzer literarischer Texte ist eine Konfrontation mit den zu übersetzenden idiomatischen Spracheinheiten unvermeidbar. Für diese Fälle bietet die übersetzungswissenschaftliche Literatur Methoden an, von denen der Übersetzer je nach Bedarf/Fall Nutzen ziehen kann.

Koller (2004) erwähnt, dass die Sprache bei der Wirklichkeitserfassung und der Interpretation dieser eine relevante Rolle spielt. „In ihr schlagen sich die Wirklichkeitsinterpretationen nieder, die in einer Kultur (in einem kommunikativen Zusammenhang) gelten. Wo diese Wirklichkeitsinterpretationen voneinander abweichen, stellt sich zugleich das Problem der Übersetzbarkeit“ (ebd. s. 172). Diese Behauptung findet u.a. auch bei Georgios Floros (2003) Zustimmung. 
„Diejenigen Elemente der ausgangssprachlichen kulturellen Konstellationen, die sich als mit den entsprechenden zielsprachlichen Kultursystemen inkompatibel erwiesen haben (im Fall einer Teil- bzw. Nicht- Identität von (Sub-) Holemen bei den entsprechenden ausgangssprachlichen und zielsprachlichen Kultursystemen), stellen die kulturspezifischen Fälle dar“ (s. 85).

Nach Koller (vgl. 2004, s. 228) lassen sich im lexikalischen Bereich fünf Entsprechungstypen unterscheiden: Davon werden hier die Eins-zu-eins-, Eins zu viele-, Eins zu Null Entsprechungen und das kommentierende Übersetzungsverfahren behandelt.

Die folgende türkische Redewendung und deren Übersetzung liefert uns ein interessantes Beispiel dafür, dass es trotz unterschiedlicher Kultur und Sprache; Türkisch-Deutsch, eine totale Äquivalenz bzw. eine Eins-zu-eins Entsprechung der Redewendungen vorliegt.

\section{Beispiel 1}

Originalwerk “72.Koğuş“ (1952: Übersetzung „72. Zelle“ (2009: 60) Übersetzungsverfahren

36)

"Burnunu sokmadığı şey yoh, „Glaub mir, es gibt nichts, in das er Eins zu eins Entsprechung babam.” nicht seine Nase reinsteckt; [...]”

„Neugierig sein“ oder „sich in eine Sache einmischen“ findet imDeutschen ihren Ausdruck in der Redensart „die Nase in etwas (rein)stecken“, im Türkischen in „burnunu sokmak“. Eine Redewendung in einer Sprache weist in Hinsicht auf die Lexik, Syntax, Semantik und im Gebrauch eine Gemeinsamkeit in einer anderen Sprache auf. Die Redewendung imTürkischen hat eine Eins-zu-eins-Entsprechung im Deutschen. Dieses Beispiel führt zu dem Schluss, dass es bei einer Eins-zu-eins-Entsprechung auf die ausgangssprachlichen idiomatischen Ausdrücke an kommt, für die unter den Ausdrücken in der Zielsprache eine Eins-zu-eins Entsprechung $\mathrm{zu}$ finden ist. In diesen Fällen gelingt die Übersetzung ohne Schwierigkeiten. Es zeigen sich auch Beispiele, die darstellen, dass der Ausdruck gleicher Begebenheiten semantisch übereinstimmend sind, sich jedoch hinsichtlich ihrer Lexik unterscheiden können. Dazu das folgende Beispiel, das zeigt, dass der Übersetzer eine Entsprechung unter vielen; Eins zu viele Entsprechung gewählt hat.

\section{Beispiel 2}

\section{Originalwerk “72.Koğuş“ (2003: Übersetzung „72. Zelle“ (2010: 34- Übersetzungsverfahren 26) 35) \\ "Kaptan için canımı veririm." "Für den Kapitän würde ich meine Eins zu viele Entsprechung Hand ins Feuer legen."}

Hier trifft der Übersetzer die freie Entscheidung und wählt für die idiomatische Wendung ,canını vermek“, „die Hand ins Feuer legen“, was im Deutschen Äquivalente wie "die Garantie übernehmen" , " Brief und Siegel geben " oder "bürgen" hat. Der Gebrauch des Idioms ist identisch. In diesem Fall erwartet den Übersetzer kein Problem.

„Übersetzungen kommen in der Regel nicht ohne kommentierende [...] Verfahren aus, wenn sie bestimmte Werte des AS-Textes dem zielsprachigen Leser vermitteln [...] sollen (Koller, 2004, s. 267), 
wenn von 1:0 Entsprechung die Rede ist. In diesem Fall ist der Übersetzer dazu gezwungen, das im Originaltext Gesagte mittels seines Kommentars in die Zielsprache zu vermitteln.

\section{Beispiel 3}

Originalwerk “72.Koğuş“ (2003: Übersetzung „72. Zelle“ (2010: 9) Übersetzungsverfahren

5)

"Zarımı kesip durmasana "Hör auf damit, du brichst mir die Kommentierendes

haybeci!" Treffer stückweise weg, du Verfahren

Abzocker!"

\section{Eine Analyse der falschen Übersetzungen am Beispiel von "72. Zelle"}

Als potentielle Fehlerquellen gelten u.a. lexikalisch-semantische Fehler, außersprachliche Fehler; die durch Stress, Ermüdung, Zeitmangel des Übersetzers zustande kommen, Kompetenzfehler oder das Zusammenwirken mehrerer Ursachen. Darunter können auch einige Fehler genannt werden, für die sich keine bestimmte Ursache beschreiben lässt. Diese können Flüchtigkeitsfehler, durch Konzentrationsmangel oder durch die Unachtsamkeit des Übersetzers hervorgekommene Fehler sein. Hier geht es um die Feststellung der Ursachen der Fehler und die daraus entstehenden Fehlertypen.

„Je stärker die kommunikativen Zusammenhänge voneinander abweichen, umso größer ist die kommunikative Herausforderung für den Übersetzer, der diese kommunikative Differenz überbrücken muss „(Koller, 2011, s. 55). Auch von Paul Kußmaul (2010) wird auf die Bedeutung des Kultur-Sprache Verhältnisses hingewiesen: „Wörter existieren nicht im leeren Luftraum“ (s. 41), und ein Text ist in eine Situation und diese wiederum in eine Kultur eingebettet. Der Übersetzer sollte in Situationen, in denen sich Ausgangs- und Zielsituation und Ausgangs- und Zielkulturen nicht überschneiden, besonders nachdenklich handeln (vgl. Kußmaul, 2010, s. 53). Wie Recht er behält, indem er dem Übersetzer diesen Hinweis nahelegt, macht sich an einigen Stellen in der Übersetzung der „72. Zelle“ deutlich. An den folgenden Beispielen wird ersichtlich, dass durch dieselbe Ursache verschiedene Fehlertypen entstehen können, da bei der Übertragung verschiedene Verfahren vorgezogen werden.

\section{Beispiel 4}

\begin{tabular}{ll}
\hline Originalwerk „,72.Koğuş“ (2003: 5) & "Başını iki yana salladı, lahavle çekti." \\
Übersetzung „72. Zelle“ (2010: 9) & $\begin{array}{l}\text { "[...] und wog den Kopf, gerade so, [...], als ob er Gott um eine } \\
\text { neue Chance bäte. " }\end{array}$ \\
Übersetzungsverfahren: & kommentierendes Verfahren \\
Fehlertyp: & Lexemwahl \\
Ursache: & mangelnde Sprachkompetenz \\
Korrekturvorschlag: & "[...], betete Gott um Geduld.“
\end{tabular}

Aus dieser Übersetzung des Idioms ergibt sich die Vorstellung, als ob die Figur eine gegebene Chance verpasst hätte und nun Gott um eine neue beten würde. Aus dem Kontext aber ist zu erschließen, dass die Figur das Gebet „lahavle” spricht und damit Gott um Geduld bittet. 


\section{Beispiel 5}

\begin{tabular}{ll}
\hline Originalwerk „72.Koğuş“ (2003: 9) & $\begin{array}{l}\text { "Yıllarca önce babasını liman kahvesinden çıkarken vuranların } \\
\text { amca oğullarını vurmuş, yüzünü olsun tanımadığ babasının } \\
\text { öcünü almıştı." }\end{array}$ \\
Übersetzung „,72. Zelle“ (2010: 14) & $\begin{array}{l}\text { "Der Onkel hatte diese Bluttat in Auftrag gegeben. } \\
\text { Daraufhin hatte der Kapitän zur Pistole gegriffen und eine } \\
\text { ganze Ladung auf den Cousin abgefeuert. Mit dem Racheakt } \\
\text { für einen Vater, den der Kapitän nie zu Gesicht bekommen } \\
\text { hatte, war der Fall für ihn erledigt gewesen." } \\
\text { kommentierendes Verfahren }\end{array}$ \\
Übersetzungsverfahren: & nicht sinngemäße Übersetzung \\
Fehlertyp: & mangelnde Sprachkompetenz \\
Ursache: & „Vor Jahren hatte er die Cousins des Mördes seines Vaters, der \\
Korrekturvorschlag: & gerade aus dem Hafencafé herauskam, erschossen,[...]
\end{tabular}

An diesem Beispiel ist klar zu erkennen, dass die Szene von dem Übersetzer falsch rezipiert worden ist und ihn zu einer falschen Vorstellung geführt hat, was wiederum eine falsche Übersetzung zur Folge hatte. Kußmaul (2010) "meint, dass es wichtig ist, “[...] (s. 68), den Kontext auf sich wirken zu lassen. Er löst bei uns die szenischen Vorstellungen aus, mit Hilfe derer wir entscheiden können, wie genau wir übersetzen wollen.” Denn wie an folgenden Beispielen klar zu sehen ist, lässt sich der Übersetzer den Kontext nicht auf sich wirken. Löst sich dieser beim Übersetzer keine szenische Vorstellung aus, ist der Übersetzer selber verwirrt, wirkt dies auf den Leser der Zielsprache. Ein Weg, mit den für den Übersetzer kompliziert erscheinenden Kontexten klarzukommen könnte es sein, die Sätze grammatisch zu strukturieren; Antworten auf die Fragen: wer, wen, was, zu finden.

Kimi vurdu: $\quad$ babasını liman kahvesinden çıkarken vuranların amca oğullarını

Wen abgeschossen: die Cousins der Täter des Mordes an seinem Vater

Der Satz „Der Onkel hatte diese Bluttat in Auftrag gegeben“, ist ein falscher Kommentar des Übersetzers und „unnötig“, denn der Übersetzer selbst scheint konfus zu sein und versucht durch eine Erläuterung eine gedankliche Ordnung für den Zielsprachenleser zu schaffen. Auf den Inhalt bezogen, ändert dieser Fehler den Sinn des Inhalts. Durch diesen Fehler ist zwar die Verständlichkeit der Aussage nicht beeinträchtigt aber der Übersetzer ist dem Originaltext „nicht treu“ geblieben.

Ein Beispiel von gleicher Art sieht man in der folgenden Übersetzung: 


\section{Beispiel 6}

\begin{tabular}{ll}
\hline Originalwerk „72.Koğuş“ (2003: 18) & $\begin{array}{l}\text { "Babasının kanını dökenlerin amca oğulları kahvedeydiler, } \\
\text { sarhoştular, [...]" }\end{array}$ \\
Übersetzung „72. Zelle“ (2010: 24) & $\begin{array}{l}\text { "Die Söhne seien die Täter, hieß es, seine eigenen Cousins. } \\
\text { Der Käpt'n erwischte sie in betrunkenem Zustand." }\end{array}$ \\
Übersetzungsverfahren: & kommentierendes Verfahren \\
Fehlertyp: & Unberechtigter subjektwechsel \\
Ursache: & mangelnde Sprachkompetenz \\
Korrekturvorschlag: & „Die Cousins des Mörders, der das Blut seines Vaters \\
& vergossen hatte, saßen im Café, sie waren betrunken [...]" \\
\hline
\end{tabular}

„Blutsrache ausüben“ oder „blutige Rache nehmen“ ist ein Verfahren, das heute noch in manchen Völkern praktiziert wird. Die engen Verwandten, meistens die männlichen Angehörige eines duch Mord Verstorbenen, sind dazu verpflichtet, seine Seelenruhe herzustellen, indem sie seinen Tod rächen. Blutrache wird im Duden online wie folgt beschrieben: „Form der Selbstjustiz, bei der ein getöteter Sippenangehöriger an dem Mörder oder einem Mitglied von dessen Sippe gerächt wird”. Da der Kapitän genau aus diesem Anlass im Gefängnis sitzt, ist es wichtig, dem Leser diesen Grund treu zu vermitteln, aber genau hier scheint die Sprachkompetenz des Übersetzers am Kontext zu scheitern. Denn er bringt den Leser in Unklarheit. Der Satz „Die Söhne seien die Täter, hie $\beta$ es, seine eigenen Cousins“, sagt weder über die Situation etwas, noch über die Gestalten in dem Originalwerk. Hier kommentiert der Übersetzer die Handlung, um den Leser zu klären, versucht aber anscheinend seine Unsicherheit zu verbergen. Die Hinweise dazu liefern die inhaltsbezogene Erklärungsversuche.

\section{Beispiel 7}

\begin{tabular}{ll}
\hline Originalwerk „72.Koğuş“ (2003: 40) & "Ciğerleri iki para etseydi bari pislerin! " \\
Übersetzung „,72. Zelle“ (2010: 50) & $\begin{array}{l}\text { "Nicht noch einmal sollte der Kapitän für die Leber dieser } \\
\text { Dreckskerle ein paar Münzen ausgeben. " }\end{array}$ \\
Übersetzungsverfahren: & Wort-für-Wort-Übersetzung \\
Fehlertyp: & Idiomkenntnisbezogen \\
Ursache: & mangelnde Sprachkompetenz \\
Korrekturvorschlag: & “Wenn die einen Dreck wert wären, diese Schmutzkerle”! \\
\hline
\end{tabular}

„Ciğer(le)i iki para etmemek“ ist eine Redensart und wahrscheinlich vom Übersetzer als solche nicht erkannt worden. Eine Wort-für-Wort-Übersetzung ergibt in der Zielsprache keine gleichwertige Entsprechung. Im Türkischen bedeutet diese Redensart „nicht tauglich, nichts wert“, oder „unzuverlässig sein“. „,... für die Leber dieser Dreckskerle ein paar Münzen ausgeben“ ergibt im Deutschen keine sinnvolle Bedeutung. 
Ein unbezweifelbares Beispiel für die mangelnde Sprachkompetenz bietet die folgende Übersetzung:

\section{Beispiel 8}

\begin{tabular}{|c|c|}
\hline \multirow{3}{*}{$\begin{array}{l}\text { Originalwerk „72.Koğuş“ (2003: 39- } \\
\text { 40) }\end{array}$} & "Başka ağalar olsa..." \\
\hline & "Fakir fukaraya tencere mi kaynatır?" \\
\hline & "Kurban ederler! " \\
\hline \multirow[t]{2}{*}{ Übersetzung „,72. Zelle” (2010: 49) } & $\begin{array}{l}\text { "Würde dieser und jener für Arme und Geschundene Speisen } \\
\text { ausgeben?" }\end{array}$ \\
\hline & "Nein, diese hätten sie geschlachtet als Opferlämmer." \\
\hline Übersetzungsverfahren: & Wort-für-Wort-Übersetzung \\
\hline Fehlertyp: & Idiomkenntnisbezogen \\
\hline Ursache: & mangelnde Sprachkompetenz \\
\hline Korrekturvorschlag: & „ Die würden uns nicht die Butter auf dem Brot gönnen!“ \\
\hline
\end{tabular}

Der fehlerauslösende Faktor ist an diesem Beispiel idiomkenntnisbezogen, der auf die mangelnde Sprachkompetenz des Übersetzers zurückzuführen ist. „Birine bir şeyi kurban etmek“ bedeutet im Deutschen ,jemandem nicht die Butter auf dem Brot gönnen“. Hier wäre eine Alternative Übersetzung einfach: „Nie würden sie uns das gönnen“ auch möglich.

Interessanter wird es bei Wort-für-Wort-Übersetzungen bei der der Übersetzer den Sinngehalt “ nicht auf der Basis einer lexikalischen oder syntagmatisch-syntaktischen Eins zu Eins Entsprechung wiedergeben kann, ohne gegen -im weitesten Sinne- idiomatische Gebrauchsbedingungen der ZS zu verstoßen“ (Wills, 1992, s. 74). Demnach signalisieren die folgenden Beispiele einen offenen Versto $\beta$ gegen die Anwendung der Idiome.

Das, was hier im Folgenden vorgeführt wird, sind klare Beispiele dafür, dass idiomatische Wendungen „nicht als Summe der Bedeutungen der Einzelelemente beschreibbar sind“.

\section{Beispiel 9}

\begin{tabular}{ll}
\hline Originalwerk „72.Koğuş“(2003: 23)) & $\begin{array}{l}\text { "Ben diyorum bayram haftası, sen diyorsun mangal } \\
\text { tahtası!" }\end{array}$ \\
Übersetzung „72. Zelle” (2010: 31) & $\begin{array}{l}\text { "Ich sage, die Festwochen sind angebrochen, und du sagst, } \\
\text { mach' Zündholz für den Kohleherd daraus." }\end{array}$ \\
Übersetzungsverfahren: & Wort-für-Wort-Übersetzung \\
Fehlertyp: & falsche Kollokation (Idiomkenntnisbezogen) \\
Ursache: & mangelnde Sprachkompetenz \\
Korrekturvorschlag: & „Du verstehst eh nur Bahnhof.“ \\
\hline
\end{tabular}




\section{Beispiel 10}

\begin{tabular}{ll}
\hline Originalwerk „72.Koğuş“ (2003: 30) & "Gezdiği Antep, yediği pekmez. " \\
Übersetzung „72. Zelle” (2010: 37) & $\begin{array}{l}\text { "Er treibt sich rum in Antep und sucht nach der } \\
\text { Traubenmelasse, sagt man, [...]" }\end{array}$ \\
Übersetzungsverfahren: & Wort-für-Wort-Übersetzung \\
Fehlertyp: & falsche Kollokation (Idiomkenntnisbezogen) \\
Ursache: & mangelnde Sprachkompetenz \\
Korrekturvorschlag: & Er hat keine blasse Ahnung von irgendetwas.“ \\
\hline
\end{tabular}

\section{Beispiel 11}

\begin{tabular}{ll}
\hline Originalwerk „,72.Koğuş“ (2003: 29) & "İnsan parayı paraya vermeli araya değil." \\
Übersetzung „,72. Zelle“ (2010: 36) & $\begin{array}{l}\text { "Das Geld muss man für das Glück ausgeben, nicht für die } \\
\text { Lücken!" }\end{array}$ \\
Übersetzungsverfahren: & Wort-für-Wort-Übersetzung \\
Fehlertyp: & falsche Kollokation (Idiomkenntnisbezogen) \\
Ursache: & mangelnde Sprachkompetenz \\
Korrekturvorschlag: & „Man sollte sein Geld in Geld anlegen und nicht auf die Straße \\
& werfen.”
\end{tabular}

„Wir übersetzen keine Wörter, sondern Bedeutungen "betont $\mathrm{Ku} \beta$ maul $(2010$, s. 28). Hier ist die Relation der Wörter unter semantischen Gesichtspunkten zu betrachten, da es sich um Idiome handelt. „Diese festen Kombinationen gilt es auch beim Übersetzen zu beachten, wenn wir nicht Gefahr laufen wollen, vom Leser sprachlich als nicht kompetent beurteilt zu werden“ (ebd. s. 28), meint Kußmaul und der Eindruck sprachlicher Inkompetenz würde sich nach Kußmaul natürlich negativ auf die intendierte Funktion einer Übersetzung auswirken.

Es ist nicht schwer zu erkennen, dass der Fehler bei den Übersetzungen Beispiel 9, 10 und 11 im Sprachenmangel des Übersetzers liegt. Der Sinn der Idiome wurde nicht erfasst. Eine Lösung zu den oben angegebenen Problemen wäre durch die Durchführung einer einfachen Recherche im Internet möglich. Wörterbücher oder Muttersprachler hätten zu Rate gezogen werden können. Denn die Faktoren, die die Fehler auslösen, sind in jedem Fall dieselben. In vielen Fällen bieten sich in dem Text selbst oft viele hinweisende Anzeichen für aufmerksame, konzentrierte Augen. Die Häufigkeit demonstriert, dass der Übersetzer kein Bedürfnis nach einer Recherche gefühlt hat. Wenn für diese Idiome in der Zielsprache keine Entsprechungen zu finden sind, wäre das kommentierende Verfahren eine Lösung.

Die Übersetzung eines literarischen Werks verlangt äußerste Präzision. “Übersetzung ist in einem weiteren Sinne immer Kulturarbeit, in einem engeren Sinne Spracharbeit: Arbeit mit der anderen und an der eigenen Kultur, Arbeit mit und an der eigenen Sprache” (Koller, 2011, s. 54).

Hier muss man sein Hauptaugenmerk auf die Richtung der Übersetzung richten; der Übersetzer übersetzt von der Fremdsprache in die Muttersprache. Es wird also aus der Fremdsprache in die 
Muttersprache übersetzt. Wenn es keine Eins-zu-Eins Entsprechung für das Idiom zu finden ist, das heißt; eine Eins-Zu-Null- Entsprechung der Fall ist, weil ,[...] die Systeme der konnotativen Werte, die stilprägend sind, sich in verschiedenen Sprachen nicht eins zu eins decken” (Koller, 2004, s. 242), ergibt dafür eine Wort-für-Wort-Übersetzung nicht die äquivalente Entsprechung. Koller vertritt dazu folgende Meinung:

„Die Entscheidung für eine bestimmte Entsprechung hängt einerseits von den zur Verfügung stehenden sprachlich-stilistischen (Wahl-)Möglichkeiten ab, andererseits von der Hierarchie der zu erhaltenden Werte, die der Übersetzer aus der für den betreffenden Text/die Textstelle maßgeblichen Hierarchie der Äquivalenzforderungen ableitet“ (ebd. s. 242).

Eine Übersetzung wie: "Ich sage, die Festwochen sind angebrochen, und du sagst, mach' Zündholz für den Kohleherd daraus", sagt dem Leser der Zielsprache nichts. Er verwirrt oder entfremdet den Leser. Das ist wohl der Punkt, wo Koller (1992) von dem Übersetzer „Nach- oder Neuschöpfung“ (s. 285) erwartet.

"Man erwartet von einer "gelungenen" Übersetzung, dass sie dem Leser genauso verständlich wird, wie es der Originaltext dem Originalleser ist. Ebenso sollte die Übersetzung im neuen Kontext dieselben Wirkungen und Vorstellungen hervorrufen, die es der Originaltext erreicht”' (Başarmış, 2016, s. 36).

Man beansprucht im Grunde von dem Übersetzer keine „ideale“ oder „richtige“ Übersetzung, aber dem Übersetzer müsste bewusst sein, dass durch das Übertragen der Wörter in die Zielsprache als Summe keine strukturelle Identität zu erreichen ist. Durch dieses Verfahren entsteht beim Zielsprachenleser eine Lücke und der künstlerische Charakter des Originalwerks geht völlig verloren.

\section{Schlussbemerkung}

Es war ein Anliegen dieser vorliegenden Untersuchung, anhand der Übersetzungen den Fragen nachzugehen, welche Bereiche sich in den literarischen Übersetzungen als Problembereiche aufzeigen, also als Barrieren definiert werden können und welche Verfahren als Überwindungsmöglichkeiten dieser Barrieren angewendet worden sind. Welche Kompetenzen ein Übersetzer, der einen literarischen Text übersetzt, zur Verfügung haben sollte, war eine andere Frage, nach deren Hintergründen nachgegangen worden ist. Bei der Übersetzung der idiomatischen Wendungen bereiten dem Übersetzer besonders die konnotativen Werte eines literarischen Werks Schwierigkeiten. Beispiele zeigen, dass der Übersetzer Fehler macht, wenn es ihm an sprachlicher Kompetenz mangelt.

Wenn phraseologische Einheiten wortwörtlich übersetzt werden, ergibt die Übertragung für den Leser der Zielsprache keinen Sinn, weil das Gemeinte, das Wesen, der kulturelle Hintergrund in Verlust geraten ist und an die Stelle etwas Unverständliches, Fremdes, Unbekanntes aufgetreten ist. In seinem Beitrag „Übersetzungsproblematik bei türkischen und deutschen somatischen Phraseologismen“ charakterisiert Özbay (2011) die idiomatischen Wendungen, bzw. die Phraseologismen als komplexe, lexikalische und semantische Zusammensetzungen, bei deren Entstehung eine Reihe gesellschaftsspezifische, soziokulturelle, landeskundliche und geschichtliche Fakten zu berücksichtigen sind und akzentuiert, dass bei der Entstehung eines Phraseologismus Normen und Erfahrungen des sozialen, kulturellen und sogar des wirtschaftlichen Lebens eine mitwirkende Rolle spielen (vgl. s.71). Ohne diesen Zusammenhang zu berücksichtigen, scheint es nicht möglich, idiomatische Wendungen Wort- für-Wort zu übersetzen, wenn sich in der Ausgangs- und der Zielsprache strukturell und kulturell weniger Ähnlichkeiten aufweisen, oder sogar beide Sprachen stark voneinander abweichen. Anhand der Beispiele sind zu erkennen, dass die Wortfür-Wort-Übersetzung in kultureller Hinsicht in vielen Fällen verfremdend wirkt. Wenn Wirkungsgleichheit erstrebt wird, werden demnach zielsprachliche Normen nicht erfüllt. Der Übersetzer hat zu beachten, dass er nicht eine einzig sprachliche Brücke zwischen dem Leser des Originaltextes und dem Leser des Zielsprachentextes aufbaut, sondern dass eine Gleichwertigkeit zwischen der resultierenden Wirkung der Übersetzung und des Originals erschaffen werden sollte.

Durch die Fehleranalyse wurde der Frage nachgegangen, wie es überhaupt möglich sein kann, dass es einem Übersetzer, der aus der Fremdsprache in seine Muttersprache übersetzt, nicht bewusst wird, dass 
bei der Wort-für-Wort-Übersetzung der idiomatischen Wendungen keine strukturelle Identität zu erreichen ist, dass durch dieses Verfahren beim Zielsprachenleser eine Lücke entsteht und der künstlerische Charakter des Originalwerks völlig verloren geht. Es lässt sich feststellen, dass es sich hierbei überwiegend um Fehler, die die mangelnde Sprachkompetenz verursacht $\mathrm{zu}$ haben scheint. Andere Erklärungen wären ausgeschlossen.

In dem Werk ,72. Koğuş“von Orhan Kemal liegt die Pointe gerade bei der Sprache der Figuren, die dadurch charakterisiert sind, dass sie extrem viele Phraseologismen verwenden. Da es bei literarischen Texten darum geht, neben der sprachlichen Form besonders die semantischen und ästhetischen Werte zu vermitteln, ist von einer idiomatischen Übersetzung auch eine denotativ und stilistisch äquivalente $\mathrm{zu}$ erwarten. Zumal ein gewisses Ma $\beta$ an Verlust nicht zu vermeiden ist, sollte der Übersetzer den Mangel an entscheidenden ästhetischen Qualitäten möglichst gering halten. Es wird heute noch darüber diskutiert, ob und wie sich eine Äquivalenzbeziehung zwischen dem Ausgangstext und dem Zielsprachentext herstellen lässt. Denn der Übersetzer strebt mit der Absicht, Literatur für andere Kulturen zugänglich zu machen. Problembereiche müssen festgestellt und Strategien müssen noch weiterentwickelt werden.

\section{Literaturverzeichnis}

Altan, N. (2003). Bilgisayar terimleri ansiklopedik sözlüğü. (3. bs.). Ankara: Sistem Yayınc1lık.

Başarmış, Y. (2016). Das problem der übersetzbarkeit der idiomatischen ausdrücke in den werken "72.Zelle" und "Murtaza" von Orhan Kemal. Institut für Sozialwissenschaften, Ankara: Hacettepe Universität Alman Dili ve Edebiyat1.

Bergen, I. S. (2006). Zum 'Zweck’ im recht und in der übersetzungswissenschaft. Im Carmen Heine, Klaus Schubert und Heidrun Gerzymisch-Arbogast (Hrgs.), Jahrbuch übersetzen und dolmetschen/Text and translation: Theory and methodology of translation. Gunter Narr Verlag.

Burger, H. U.a. (Hrsg.). (1982). Handbuch der phraseologie. Berlin-New York: De Gruyter.

Engberg, J. (1993). Hermes. Journal of Linguistics,10. Abgerufen von http://download2.hermes.asb.dk/archive/download/H10_09.

Floros, G. (2003). Kulturelle konstellationen in texten: Zur beschreibung und übersetzung von kultur in texten. Gunter Narr Verlag

Hennecke, A. (2009). Zum Transfer kulturspezifischer Textbedeutungen. Theoretische und methodische Überlegungen aus einer semiotischen Perspektive. Linguistik Online, 37. Abgerufen von http://www.linguistik-online.de/37_09/hennecke.html

Koller, W. (2011). Übersetzung unter den aspekten des kultur- und des sprachkontaks. (8. Aufl.) Tübingen und Basel: A. Francke Verlag.

Koller, W. (2004). Einführung in die Übersetzungswissenschaft. (7. Aufl.). Wiebelsheim: Quelle\&Meyer Verlag.

Kopetzki, A. (2015). Praxis und Theorie des literarischen Übersetzens: Neue Perspektiven. Im A. Buschmann (Hrsg.), Gutes Übersetzen: Neue Perspektiven für Theorie und Praxis des Literaturübersetzens.

Kurbanoğlu, S. S. (2004). Kaynak gösterme el kitabı. Ankara: Üniversite ve Araştırma Kütüphanecileri Derneği

Kußmaul, P. (2010). Verstehen und Übersetzen: Ein Lehr- und Arbeitsbuch. (2. Aufl.) Narr Studienbücher

Levý, L. und U.a. (1969) (Hrsg.). Die literarische übersetzung: Theorie einer Kunstgattung. Scientia Traductionis, 11. Frankfurt am Main: Athenäum Verlag.

Nord, C. (2002). Fertigkeit übersetzen. Ein selbstlernkurs zum übersetzenlernen und übersetzenlehren. Alicante: Editorial Club Universitario

O'Neil, J. M., ve Egan, J. (1992). Men's and women's gender role journeys: Metaphor for healing, transition, and transformation. B. R. Wainrib (Ed.), Gender issues across the life cycle içinde (ss. 107-123). New York, NY: Springer.

Özbay, R. (2011). Übersetzungsproblematik bei türkischen und deutschen somatischen Phraseologismen. Firat Üniversitesi Sosyal Bilimler Dergisi, 21(2), 70-86

Özbay, R. (2010). Uluslararast Klbris Üniversitesi folklor/edebiyat dergisi, 16(64), 2010/4

Frank, A. P. (Hrsg.). (1993). Göttinger Beiträge zur Internationalen Übersetzungsforschung. Im Übersetzen, verstehen, Brücken bauen: Geisteswissenschaftlichen und literarisches Übersetzen in internationalen Kulturaustausch. Berlin: Erich Schmidt Verlag. 
Redhouse çă̆daş Türkçe-İngilizce sözlüğü. (1985). İstanbul: Redhouse.

Rothfuss, Uli (2005). Abgerufen von http://www.orhankemal.org/links/175.htm

Sağlam, M. Y. (2014). Dünya edebiyatına uzanan yolda bir Türk romancı: Orhan Kemal. Erdem-İnsan ve Toplumbilim Dergisi, 87-103.

Sandrini P. (1993). Die rolle des Uübersetzers im mehrsprachigen umfeld: Zum berufsprofil des Uübersetzers im multikulturellen umfeld am beispiel südtirols. 38, 54-56

Uçak, N. (1999). Sosyal ve insan bilimleri alanlarında bilgi gereksinimi ve kullanımı. Hacettepe Üniversitesi Edebiyat Fakültesi Dergisi, 16 (1), 115-128.

Wills, W. (2004). Übersetzung als wissensbasierte tätigkeit:Übersetzung-translation - traduction. 1. teilband. (Hrsg.) A. P. Frank , Harald Kittel,Norbert Greiner,Theo Hermans,Werner Koller,Jose Lambert,Fritz Paul. Berlin-New York: De Gruyter.

Wills, W. (1992). Übersetzungsfertigkeit: Annäherungen an einen komplexen übersetzungspraktischen begriff (Tübinger Beiträge zur Linguistik). Gunter Narr Verlag.

Wordpress: Abgerufen von https://phraseologismen.wordpress.com/2011/12/29/was-sind-phraseologismen/

Zybatow, L. (2006). Translationswissenschaft: Gegenstand-methodologie-theoriebildung. Im C. Heine, K. Schubert und H. Gerzymisch-Arbogast (Hrgs.), Jahrbuch übersetzen und dolmetschen/Text and translation: Theory and methodology of translation. Gunter Narr Verlag. 